\title{
UNE TECHNIQUE DE PRÉPARATION D'EXPÉDITION ET D'UTILISATION DE CULTURES PURES DESTINÉES AUX INDUSTRIES ALIMENTAIRES UTILISANT DES LEVAINS DE MICROORGANISIMES AÉROBIES
}

par

\section{J. CLAVEAU}

Professeur à l'Ecole Nationale Supérieure des Industries Agricoles et Alimentaires

La recherche, le dénombrement, l'isolement au laboratoire des microbes aérobies mettent en ouvre des techniques classiques, utilisant un matériel varié [1], dont la caractéristique principale est d'offrir aux microorganismes une surface de culture, sur milieu approprié, largement en contact avec l'oxygène de l'air, comme les géloses nutritives réparties en boîtes de Pétri, tubes à essais inclinés ou roulés.

La culture et la propagation de ces mêmes microbes, dans le but de préparer des levains, font appel :

- Soit à des techniques identiques dans lesquelles les surfaces de culture en contact avec l'oxygène sont statiques et immobiles, milieux nutritifs gélosés ou liquides en tubes inclinés, fioles d'Erlenmeyer, fioles de Fourneau, boîtes de Legroux, boîtes de Roux par exemple.

- Soit à des techniques totalement différentes dont les surfaces de culture sont mobiles, dynamiques, en contact avec l'oxygène par un brassage continuel de la masse, par exemple : milieux nutritifs liquides en récipients de plus grande capacité avec insufflation d'air stérile ou agitation mécanique.

Avec une habileté manuelle convenable, jointe à son expérience de la pratique journalière, le microbiologiste est assuré de conduire, dans son laboratoire, ces diverses opérations, de façon irréprochable, sans la moindre souillure du milieu.

(1) Communication présentée le 13 juin 1963 à Ferrières-en-Bray, lors de l'Assemblée Générale de l'Association des Chimistes et Ingénieurs de Sucreries, Distilleries et Industries Agricoles et Alimentaires. (Industries al. et agr. 1963, no 6, 599) 
Le transport des cellules microbiennes ainsi produites, jusqu'au lieu où elles seront utilisées, peut se faire sans plus de préparation par expédition du flacon qui a servi à les propager ou, après récolte des corps microbiens par lavage, décantation, centrifugation, etc., ce qui permet l'envoi d'un volume plus réduit, mais oblige à des manipulations complémentaires qui introduisent des risques non négligeables de contamination.

Enfin, le conditionnement doit être aseptique, l'emballage robuste, le bouchage étanche ce qui n'est pas sans poser de graves problèmes dans certains cas, comme le transport de levures, où le microorganisme est fortement gazogène; pour le résoudre, l'Ecole de Brasserie de Nancy utilise un flacon en cuivre spécialement étudié, stérilisable, résistant à la pression du gaz carbonique dégagé par la levure et mettant même cette pression à profit lors de la vidange du flacon dans l'appareil à levain [2].

En résumé, la préparation par le laboratoire spécialisé d'une culture microbienne, son expédition à l'usine et son utilisation en fabrication doivent concilier trois aspects économiques et technologiques apparemment opposés.

- La qualité : état de pureté rigoureux.

- La quantité : nombre de cellules élevé.

- Le prix de revient : aussi réduit que possible (comprenant la main-d'œuvre, les flacons et emballages, et le transport aller et éventuellement retour).

La technique que nous proposons doit satisfaire à ces exigences, elle s'inspire d'une méthode simple couramment utilisée pour apprécier la qualité microbiologique du nettoyage des bouteilles dans les industries alimentaires qui conditionnent, en flacons, des liquides périssables : lait, bière, eaux minérales, jus de fruits, pour ne citer que les plus importants.

Cette méthode décrite par divers auteurs $[3,4,5,6]$ consiste à introduire dans les bouteilles, à la sortie de la laveuse, un milieu nutritif fortement gélosé, préalablement fondu au bain-marie bouillant et refroidi vers $50^{\circ}$, puis à rouler la bouteille sur elle-même, en refroidissant ses parois sous un filet d'eau de manière à faire figer le milieu gélosé en un film peu épais adhérent à la face interne de la bouteille; mise ensuite en incubation, cette dernière fera office de récipient de culture et les colonies microbiennes qui se développeront dans la gélose donneront par leur nombre, leur localisation et la nature des espèces présentes une évaluation très rigoureuse de l'efficacité du nettoyage.

Nous avons pensé opérer de façon identique, avec des bouteilles propres de 1 litre, des types [7] "Steinies» NF B 31 017, "Six 
étoiles " NF B 31020 ou "Lait stérilisé " PN B 31022 (ces dernières sont à conseiller car elles résistent mieux aux chocs thermiques), dans lesquelles on introduit $150 \mathrm{ml}$ de milieu nutritif gélosé.

Après bouchage au coton cardé, l'ensemble est stérilisé à l'autoclave durant 20 minutes à $120^{\circ} \mathrm{C}$, puis les bouteilles subissent un prérefroidissement jusqu'à $50^{\circ} \mathrm{C}$ environ, suivi d'un refroidissement énergique et rapide en position couchée, durant lequel chaque bouteille est animée d'un mouvement de rotation autour de son axe.

Elles sont enfin ensemencées avec $1 \mathrm{ml}$ d'une culture pure du microbe à propager, uniformément réparti sur la surface du film de gélose et mises en incubation.

Ces opérations successives sont schématisées sur la figure 1 .

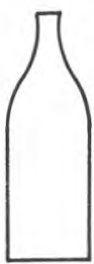

(1)

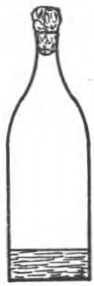

(2)

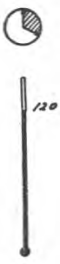

(3)

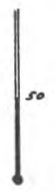

(4)

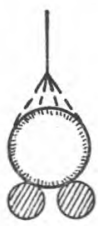

(5)

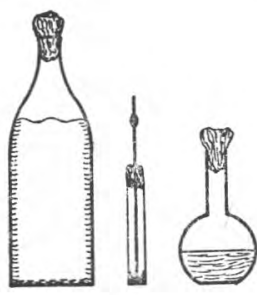

(6)

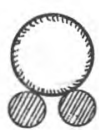

(7)

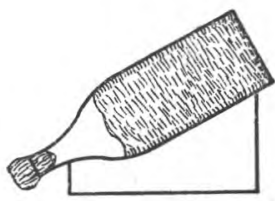

(3)

Figure 1

1. Bouteille vide et propre.

2. Répartition du milieu nutritif et bouchage.

3. Stérilisation durant 20 minutes à $120^{\circ} \mathrm{C}$.

4. Prérefroidissement vers $50^{\circ} \mathrm{C}$.

5. Roulage et refroidissement sous un filet d'eau.

6. Inoculation avec la souche microbienne.

7. Nouveau roulage pour répartir l'inoculum.

8. Incubation en position inclinée vers l'avant. 
La composition du milieu de culture, la durée et la température d'incubation varient avec l'espèce microbienne à cultiver.

La position de la bouteille, inclinée vers l'avant, facilite le renouvellement de sa micro-atmosphère, le gaz carbonique, plus lourd, s'écoule lentement et est remplacé par de l'air, filtré sur le bouchon de coton, qui apporte son oxygène à la culture.

Ces bouteilles sont expédiées sans autre bouchage que celui du tampon de coton cardé, protégé par un capuchon de papier, leur prix de revient est peu élevé, leur emballage peut être sommaire, la casse de l'une d'elles ne souille pas les autres et n'appelle pas de réclamations ou de poursuites de la part de l'organisme transporteur, leur poids est réduit, à peine supérieur à celui des bouteilles vides, les frais de transport ne sont payés qu'une seule fois le retour étant inutile.

L'utilisation de la culture microbienne par l'usine qui la reçoit peut se faire immédiatement ou dans le mois qui suit la livraison, à condition de conserver les bouteilles couchées dans un endroit frais et non humide.

Deux cas sont à envisager :

- Cas de la culture à propager.

Exemple : microorganisme : Saccharomyces cerivisiae.

Industrie utilisatrice : Brasserie.

- Cas de la culture à utiliser directement.

Exemple : microorganisme : Penicillium candidum.

Industrie utilisatrice : Fromagerie.

\section{Cas de la culture à propager}

Dans les industries qui utilisent des levains de microbes aérobies à propager, le travail de propagation se fait habituellement en plusieurs stades; les premiers, au laboratoire, préparent des quantités réduites de culture microbienne, de $10 \mathrm{ml}$ à quelques litres, puis les suivants, à l'usine, prennent le relai et peuvent en produire plusieurs hectolitres.

En brasserie, c'est le Danois Christian Hansen qui introduisit, à la fin du siècle dernier, la pratique de la propagation de la levure pure, il décrivit les opérations d'isolement des souches de Saccharomyces cerevisiae et de leur culture tant au laboratoire qu'à l'usine dans des installations ad-hoc imaginées par lui ; les détails précis de leur réalisation pratique, abondamment illustrés de photographies, 
en sont donnés dans divers ouvrages et notamment par JoRGENSEN [8] qui reproduit également un schéma de l'appareil pour cultures industrielles.

Certaines variantes de préparation furent ensuite introduites, les appareils à levain pur modernisés; nous pouvons citer parmi eux celui de Petersen et Henius repris par Hennies [9] et celui décrit par PrescotT et Dunn [10].

Le principal reproche que l'on puisse faire à toutes ces opérations successives, ainsi qu'aux appareils utilisés, est leur manque de simplicité et, de ce fait, leur conduite délicate; DE CLERCK [11] à leur propos écrit : "Ces appareils demandent beaucoup de soins et de surveillance. On peut réaliser la multiplication de levains de culture pure dans des conditions beaucoup plus simples et qui présentent autant de sécurité ". Nous partageons entièrement cette opinion et pensons que la technique que nous préconisons simplifie considérablement les manipulations.

Au moment de leur utilisation les bouteilles sont essuyées longuement avec un tampon de coton hydrophile imbibé d'eau de javel et déposées sur la table du laboratoire désinfectée de la même façon.

En observant les règles élémentaires des manipulations aseptiques, et notamment le flambage des cols à la flamme d'un bec Bunsen après chaque débouchage et avant chaque rebouchage des divers flacons, les bouteilles sont remplies jusqu'au niveau du rétrécissement du col avec un moût de bière de densité moyenne, non houblonné, correspondant à une bonne bière type "bock" à pH voisin de 4,5 et stérilisé à l'autoclave en ballons bouchés au coton cardé.

Avant le versement dans la bouteille, ce moût est amené à la température de $14^{\circ} \mathrm{C}$ et convenablement aéré par agitation $\mathrm{du}$ ballon.

Les bouteilles remplies et rebouchées sont maintenues à 12 -14 $^{\circ} \mathrm{C}$ durant 24 heures environ; pendant ce temps la culture de levure qu'elles renferment passe de l'état dormant au début de l'état proliférant, le nombre de cellules, qui est de l'ordre de $5 \times 10^{10}$ par bouteille, en est peu modifié, mais la phase d'adaptation est franchie ; si l'on est parti de 10 bouteilles, la préparation au laboratoire est terminée.

La propagation à l'usine, dans un local de dimensions réduites, tenu en parfait état de propreté et contiguë à la salle de fermentation principale, se fera à l'aide de 2 cuves très simples de 3 et 70 hectolitres environ (figure 2). 


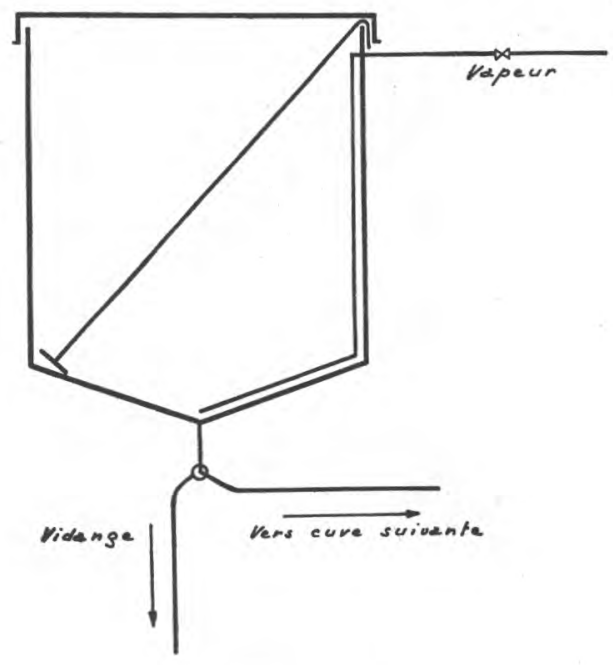

Figure 2

Ces deux cuves doivent obligatoirement satisfaire aux exigences impératives suivantes :

- être facilement nettoyées, désinfectées, rincées et vidées ;

- être stérilisables par admission prolongée d'un jet de vapeur ;

- être équipées pour recevoir un moût stérile au sens "industriel » du terme;

- être recouvertes d'un couvercle débordant à l'extérieur.

Tout autre dispositif supplémentaire, système de refroidissement, d'agitation mécanique, de barbottage d'air stérile, bien que théoriquement très utile, introduit des risques d'infection importants, alourdit considérablement la conduite de la fermentation en multipliant les manipulations et rend l'installation justiciable des critiques que nous avons antérieurement faites.

«En matière de microbiologie la règle d'or de la réussite demeure la simplicité. »

Après avoir à nouveau désinfecté les parois des bouteilles à l'aide d'un tampon de coton imprégné d'eau de javel et flambé rapidement leurs cols, chaque bouteille est versée dans la première cuve contenant 40 litres de moût non houblonné, stérile, à 12-14 $\mathrm{C}$. Le versement se fait en deux fois : la moitié du liquide non agité d'abord, afin d'éviter les débordements, puis le reste du liquide après agitation pour emporter la totalité des cellules. 
La suite de la progression des volumes peut se faire comme l'indique le diagramme de propagation (figure 3) ; 24 heures plus tard, 2 hectolitres de moût houblonné stérile refroidi à $12-14^{\circ} \mathrm{C}$ sont versés sur le pied de levain ; 24 heures plus tard, le contenu de la petite cuve est agité puis déversé dans la grande cuve contenant 10 hectolitres de moût houblonné à 12 -14 $^{\circ} \mathrm{C}$; 24 heures plus tard, la cuve reçoit un versement complémentaire de 50 hectolitres de moût houblonné à $10-12^{\circ}$, et le lendemain servira à ensemencer un bassin de $250 \mathrm{hl}$ environ.

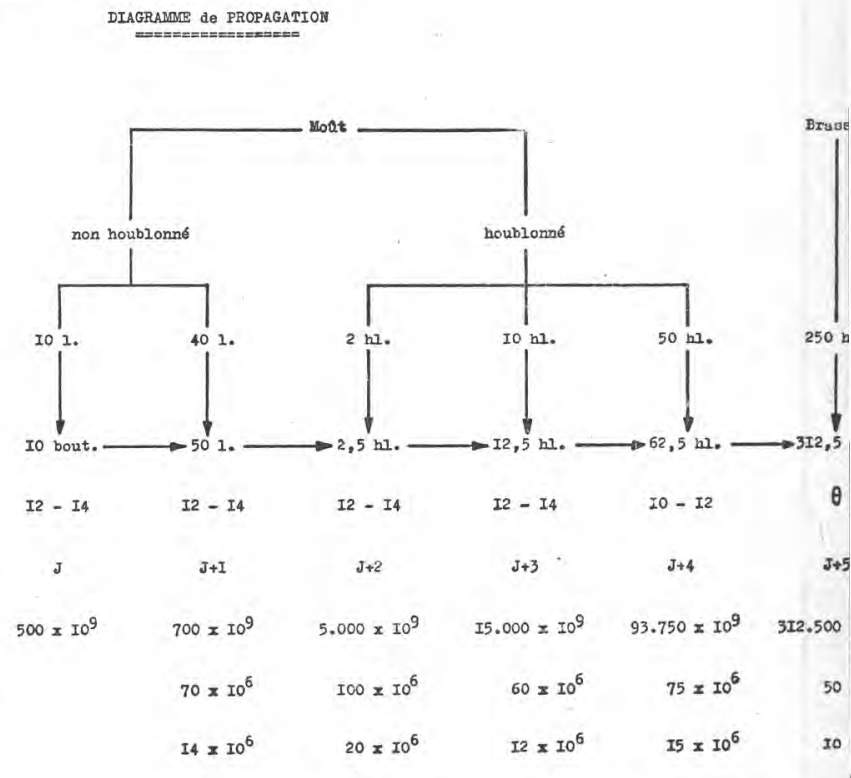

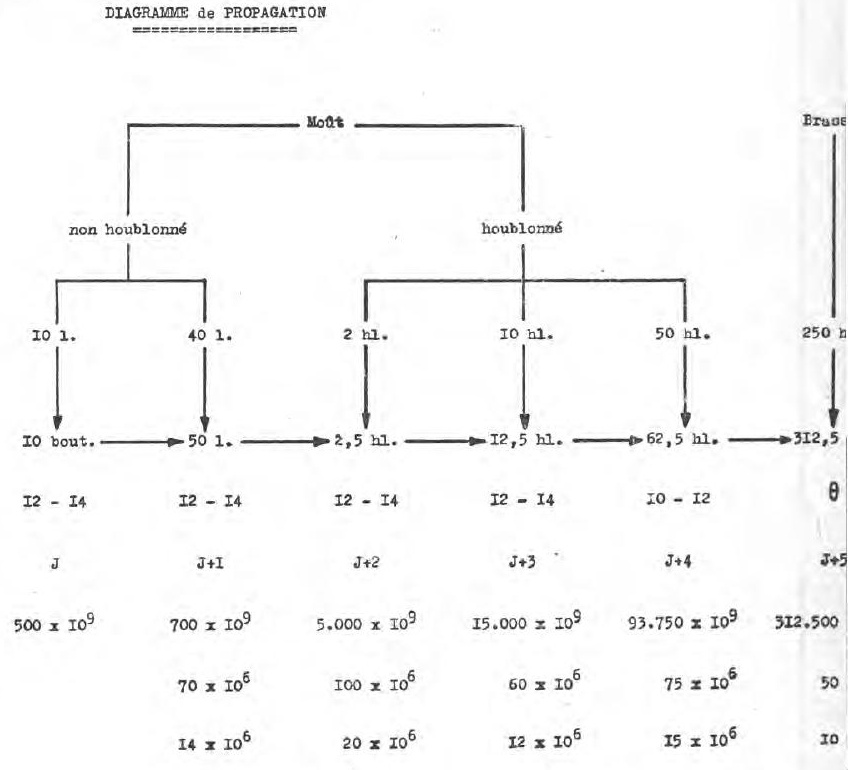

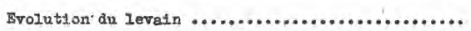

Teepérature en degrés 0

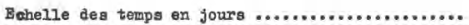

\$ombre totel de cellules

Nombre de oell.ules $/ \mathrm{ma}: . .\left\{\begin{array}{l}\text { avant versement } . . . . . \\ \text { apriss versement ....... }\end{array}\right.$

Figure 3

Bien entendu, ce diagramme est proposé à titre indicatif, il donne de bons résultats pour une levure de rapidité normale, il peut être modifié pour une levure de caractéristiques différentes, il importe surtout de toujours avoir des inoculations massives et de s'efforcer de ne jamais descendre à une concentration en cellules inférieure à 5 millions $/ \mathrm{ml}$; la surveillance quantitative de cette population s'effectuera facilement par comptage direct sous le microscope à l'aide de cellules appropriées, comme celle de MaLassEz, opération simple décrite dans tous les ouvrages techniques de microbiologie [12].

La figure 3 donne également les populations microbiennes moyennes que nous avons obtenues dans nos essais en suivant ce 
diagramme; la progression du nombre total de cellules en fonction $d u$ temps est représentée par le graphique de la figure 4, elle permet de déterminer le “temps de reproduction " moyen égal à 11 heures environ.

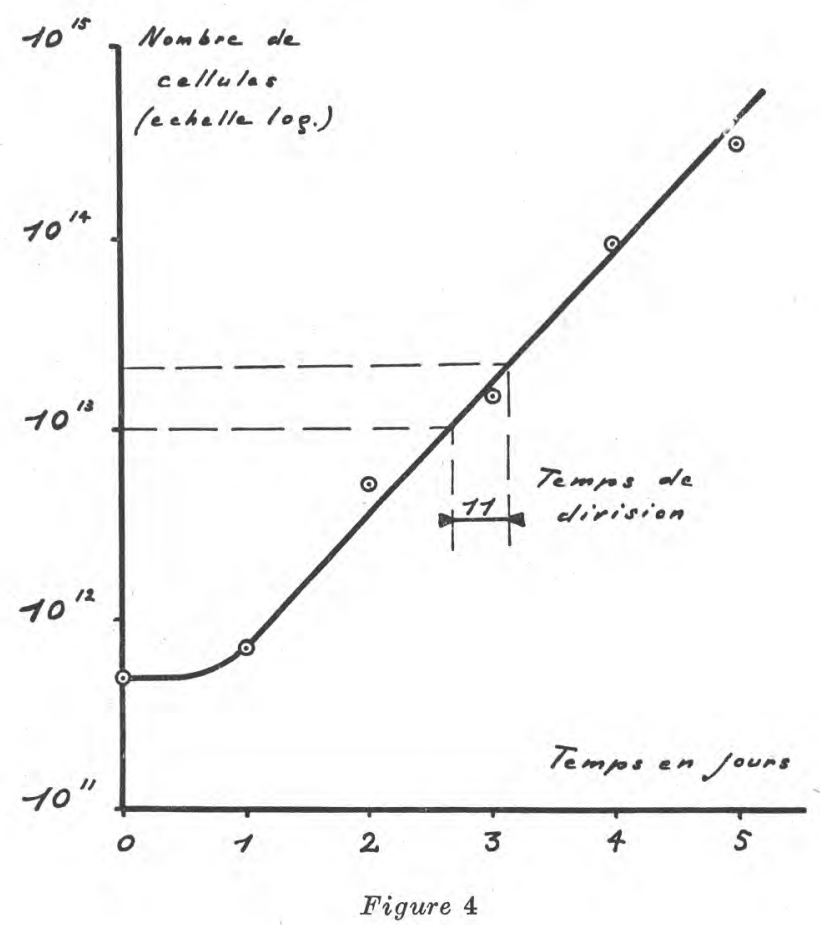

\section{Cas de la culture à utiliser directement}

Certaines industries utilisent des cultures microbiennes qu'elles emploient sans propagation préalable, tel est le cas par exemple, des fromageries de "pâtes molles à moisissures externes" qui fabriquent des fromages comme le Camembert, ensemencés en surface, après égouttage du sérum et démoulage, avec une suspension de spores d'une moisissure blanche, le Penicillium candidum.

En général, ces spores ou «conidies» sont obtenues, dans des laboratoires spécialisés, de la façon suivante [13].

Le microorganisme soigneusement isolé et choisi selon des critères qu'il serait trop long de détailler ici, est cultivé sur boîtes de Roux durant plusieurs semaines, les conidies et fragments de mycelium sont récoltés par lavages successifs avec de faibles quantités 
de liquides physiologiques stériles (souvent de l'eau salée à la concentration de $9 \mathrm{~g}$ /litre).

Le liquide de récolte est tamisé sur étamine stérile pour retenir les gros fragments mycéliens, puis, à la suite de divers traitements qui ont pour but de plus ou moins concentrer sa teneur en conidies, conditionné en flacons ou ampoules et expédié à l'usine qui, après dilution convenable, l'utilisera en pulvérisation à la surface des fromages ou en atomisation de l'atmosphère des haloirs.

Dans certains cas ces cultures sont livrées sous forme de poudres qui peuvent être remises en suspension et utilisées comme précédemment ou mélangées au sel fin et sec servant à saler les fromages ; très rarement ces poudres sont obtenues par lyophilisation.

Toutes ces opérations de culture, récolte, traitement et conditionnement doivent être conduites avec le plus grand respect de l'aseptie, elles sont de ce fait très délicates et jamais à l'abri absolu de contaminations et de souillures extérieures ; une infection, même légère, à ce stade de préparation ira toujours en s'aggravant jusqu'au moment de l'emploi, surtout s'il s'agit de suspensions aqueuses, les poudres étant plus stables.

Enfin, même si elles sont bactériologiquement irréprochables, ces préparations commerciales, dans lesquelles les organismes sont séparés de leur substrat nourricier et profondément désorganisés, ne peuvent que perdre de leur activité entre le moment de leur fabrication et celui de leur utilisation; cette perte est tout au plus réduite au minimum pour les poudres lyophilisées.

Préparées suivant la technique que nous préconisons, les cultures arrivent à l'usine sans avoir subi la moindre transformation ; durant le voyage et le stockage qui précèdera leur emploi, elles continueront à végéter sur leur milieu nutritif et, à condition de ne pas dépasser les limites raisonnables de la conservation, qui aux alentours de $15^{\circ} \mathrm{C}$ peuvent être fixées à un mois environ après la date de réception, aucune perte d'activité notable ne sera enregistrée.

$\mathrm{Au}$ moment de l'emploi, la récolte se fera par lavages successifs du feutrage mycélien avec le liquide physiologique stérilisé ou simplement bouilli, quelques billes de verre faciliteront la dilacération mécanique de la culture, l'utilisation extemporanée de la suspension évitera, au cas où elle se serait produite, la prolifération de toute infection microbienne.

Une bouteille de 1 litre développe une surface de culture de $500 \mathrm{~cm}^{2}$ environ soit plus de deux fois celle d'une boîte de Roux, elle produit à maturité une moyenne de $2 \times 10^{10}$ conidies et peut fournir plusieurs litres de suspension. 
La récolte peut également se faire par agitation rapide de la bouteille avec plusieurs portions successives de 200 à $300 \mathrm{~g}$ de sel fin bien sec qui, après homogénéisation, serviront au salage des fromages.

En conclusion, la grande simplicité de préparation, le nombre élevé de cellules produites constamment maintenues, sans perturbation, sur le substrat nourricier originel, à leur maximum de viabilité, la pureté microbiologique parfaite assurée par un minimum de manipulations, font de la présentation des cultures microbiennes aérobies en bouteilles roulées un moyen sûr et efficace de préparation des levains purs.

Le prix de revient réduit, l'emballage et le transport faciles, l'utilisation simple et échelonnée dans le temps sans risques de mortalité excessive ou de pollution dangereuse, rendent leur emploi industriel pratique, souple et sans risques.

Voilà un ensemble d'avantages qui ne manquera pas de concilier le point de vue du microbiologiste et celui du technicien; il devrait inciter l'un et l'autre sinon à adopter cette technique, du moins à l'essayer.

\section{BIBLIOGRAPHIE}

[1] P. Hadduroy. Microbiologie générale et techniques microbiologiques, Masson, Paris, 1947.

[2] E. B. N. Méthodes d'analyses pour le controble de laboratoire en Malterie et en Brasserie. Imprimeries Humblot, Nancy, 1956.

[3] L. A. Allen, S. M. Pasley et M. A. F. Pierce. J. gen. Microbiol., $1952,7,257$.

[4] R. Buttiaux. Rev. Hyg. et Med. Soc., 1959, 7, 131.

[5] R. Buttiaux. Ann. Inst. Pasteur. Lille, 1960, 11, 23.

[6] A. Boudier, F. CARdey et M. Rossiaud. Ann. Inst. Pasteur. Lille, 1960, $11,55$.

[7] P. A. Caron. Techniques modernes d'embouteillage. Compagnie française d'éditions, Paris, 1960.

[8] A. Jorgensen. Micro-organisms and fermentation. Charles Griffin and Company, London, 1948.

[9] K. Hennies. Manuel du Brasseur. Editions de "La Boisson ", Bruxelles, 1960.

[10] S. C. Prescots et C. G. Dunn. Industrial Microbiology. MeGraw-Hill, Book Company, New York, 1959.

[11] J. De Clerck. Cours de Brasserie, Université de Louvain, HeverleeLouvain, 1962.

[12] C. Jaulmes, A. Jude et J. Querangal des Essarts. Pratique du Laboratoire. Masson, Paris, 1958.

[13] R. Veisseyre. Techniques laitières modernes. Maison Rustique, Paris, 1957. 